\title{
Lecture socio-politique de l'empire : bilan et perspectives
}

A socio-political interpretation of the Empire. Review and perspectives

\section{Natalie Petiteau}

\section{(2) OpenEdition \\ 1 Journals}

Édition électronique

URL : https://journals.openedition.org/ahrf/11503

DOI : $10.4000 /$ ahrf. 11503

ISSN : 1952-403X

\section{Éditeur :}

Armand Colin, Société des études robespierristes

\section{Édition imprimée}

Date de publication : 1 janvier 2010

Pagination : 181-202

ISSN : 0003-4436

\section{Référence électronique}

Natalie Petiteau, «Lecture socio-politique de l'empire : bilan et perspectives », Annales historiques de la Révolution française [En ligne], 359 | janvier-mars 2010, mis en ligne le 01 mars 2013, consulté le 23 avril 2022. URL : http://journals.openedition.org/ahrf/11503 ; DOl : https://doi.org/10.4000/ahrf. 11503 


\section{LECTURE SOCIO-POLITIQUE DE L'EMPIRE : BILAN ET PERSPECTIVES}

Natalie PETITEAU

L'histoire socio-politique des années consulaires et impériales a été longtemps négligée, mais les renouvellements de l'historiographie, au cours des deux dernières décennies, autorisent aujourd'hui une relecture de la période. La synthèse que voici met en évidence le fait que l'on peut mieux comprendre les attitudes des Français à l'égard du régime. En prenant en compte les travaux de prosopographie et de biographie, en observant des espaces sociaux spécifiques, en interrogeant des moments clefs, on peut désormais mesurer les permanences et les innovations qui marquent la période.

Mots-clés : opinion, histoire politique, histoire sociale, Empire, souverain, oppositions, adhésions.

D'un bicentenaire à l'autre, de la célébration de la naissance de Napoléon, en $1969^{1}$, à la non commémoration de la fondation du Consulat ou de l'avènement de l'empereur, de 1999 à 2004, l'historiographie du Premier Empire a en fait considérablement mais silencieusement progressé. Les colloques organisés à Paris, par l'Institut Napoléon ${ }^{2}$, à Avignon ${ }^{3}$, puis

(1) Voir pour les publications scientifiques La France à l'époque napoléonienne, $\mathrm{n}^{\circ}$ spécial de la Revue d'histoire moderne et contemporaine, tome XVII, juillet-septembre 1970; pour les ouvrages destinés au grand public, voir la bibliographie dans Natalie Petiteau, Napoléon, de la mythologie à l'histoire, Paris, Le Seuil, 1999, p. 397-429.

(2) Jacques-Olivier Boudon (dir.), Brumaire. La prise de pouvoir de Bonaparte, Paris, éditions SPM, 2001, 180 p.; Jacques-Olivier Boudon (dir.), Armée, guerre et société à l'époque napoléonienne, Paris, éditions SPM, 2004, 257 p.; Jacques-Olivier Boudon (dir.), Le Concordat et le retour de la paix religieuse, Paris, éditions SPM, 2008, 219 p.

(3) Natalie Petiteau (dir.), Voies nouvelles pour l'histoire du Premier Empire. Territoires, pouvoirs, identités. Actes du colloque d'Avignon, 7-8 mai 2000, Paris, La Boutique de l'histoire, 2003, 302 p.

ANNALES HiSTORIQUES DE LA RÉVOLUTION FRANÇAISE - 2010 - No 1 [181 À 202] 
à La Roche-sur-Yon ${ }^{4}$, ont témoigné de cette vitalité. Celle-ci apparaît aussi au travers des bilans sur la période révolutionnaire dressés par l'UMR Telemme à Aix en $2001^{5}$, l'IHRF à Paris en $2004^{6}$, ou encore l'IRHIS à Villeneuve-d'Ascq en 2006 , car les ouvertures sur la période napoléonienne n'y ont jamais été négligées. Ces rencontres scientifiques se sont de plus déroulées alors que de nouveaux et souvent jeunes chercheurs apportaient aux débats les fruits de leurs thèses de doctorat ${ }^{8}$.

L'ensemble de ces travaux autorise aujourd'hui une lecture sociopolitique des années napoléoniennes qui était impossible en 1969 et qui est essentielle à la compréhension du XIX ${ }^{\mathrm{e}}$ siècle. Car le Premier Empire opère la transition entre l'Ancien Régime et les innovations multiples d'un $\mathrm{XIX}^{\mathrm{e}}$ siècle qui n'en finit pas de mettre en pratique les héritages de la Révolution et de l'Empire, plus ou moins rapidement selon qu'il s'agit du champ politique, social ou culturel. La compréhension du premier siècle des temps contemporains ne peut donc pas faire l'économie d'une analyse socio-politique des années 1800-1815. Les données du social comme celles du politique s'établissent alors en fonction des mutations révolutionnaires pour imposer des cadres qui demeurent efficients tout au long $\mathrm{du} \mathrm{XIX}^{\mathrm{e}}$ siècle.

En saisissant ce qu'ont été les perceptions des structures politiques du régime dans les différents espaces de la société consulaire et impériale, on mesure mieux la portée et les limites de l'œuvre révolutionnaire, on comprend mieux les réalités sociales post-révolutionnaires, on saisit mieux les nouvelles donnes de la vie politique. Il est en effet possible désormais de proposer une synthèse sur la façon dont notables, anonymes, employés en nombre croissant, travailleurs de la terre, de l'atelier ou de la manufacture ont vécu et ressenti cette mise en ordre inédite, ce régime policier, cette modernisation étatique, et cet état de guerre permanent dont les moti-

(4) Jean-Clément MARTin (dir.), Napoléon et l'Europe. Colloque de La Roche-sur-Yon, Rennes, Presses Universitaires de Rennes, 2002, 169 p.

(5) Martine Lapied, Christine Peyrard (dir.), La Révolution française au carrefour des recherches, Aix-en-Provence, Presses de 1'Université de Provence, 2003, 356 p.

(6) Jean-Clément Martin (dir.), La Révolution à l'œuvre. Perspectives actuelles dans l'histoire de la Révolution française, Rennes, Presses Universitaires de Rennes, 2005, 375 p.

(7) Jean-Pierre JESSENNE (dir.), Vers un ordre bourgeois? Révolution française et changement social, Rennes, Presses Universitaires de Rennes, 2007, 418 p.

(8) Voir ci-dessous les mentions des travaux de Louis Bergès, Walter Bruyère-Ostells, Stéphane Calvet, Pascal Chambon, Laurence Constant, Jacques Hantraye, Isabelle Laboulais-Lesage, Claire Lemercier, Aurélien Lignereux, Igor Moullier, Karine Rance, Anne Rolland-Boulestreau, Cyril Triolaire. 
vations pouvaient aisément échapper ${ }^{9}$. Autant de questions qu'il est essentiel de résoudre pour faire progresser l'histoire socio-politique d'années charnières et cruciales dans l'histoire de l'Europe contemporaine.

L'histoire sociale et les interrogations sur le politique ont suffisamment progressé au cours des dix dernières années pour permettre de tenter un bilan. En voulant clore la Révolution par une entreprise de recomposition sociale inédite, le Consulat puis l'Empire ont misé sur la maîtrise des données du social pour accomplir une conquête politique. C'est en organisant une hiérarchie nouvelle, de la Légion d'honneur à la noblesse d'Empire, que le régime napoléonien a ambitionné de séduire des citoyens redevenus finalement des sujets, c'est par une refondation du social que Napoléon s'efforce de clore très concrètement la Révolution en orchestrant une stabilisation jamais entreprise jusqu'alors. L'enjeu était d'importance compte tenu de la crise que traversait la société française depuis la fin du XVIII ${ }^{\mathrm{e}}$ siècle, crise dont elle n'était toujours pas sortie sous le Directoire. L'œuvre sociale est donc aussi stratégie politique, et c'est entre autres en examinant la portée de celle-ci que l'on peut comprendre les adhésions et les soutiens à l'Empire.

Or, des trajectoires d'individus emblématiques et de groupes sociaux spécifiques ont été reconstituées. Elles permettent une première approche de l'histoire socio-politique de l'Empire, selon différents angles de vue, loin des cadres selon lesquels ont été longtemps pensées l'histoire sociale et l'histoire politique. Seront ensuite envisagés des espaces spécifiques, du théâtre à la France rébellionnaire, du monde du commerce à l'univers militaire, où apparaissent d'autres pans essentiels de l'histoire socio-politique de la période. Il restera alors à adopter un dernier point de vue, celui des réceptions de l'Empire en des moments clefs, du sacre de Napoléon au dénouement de 1814, par exemple. Autant de pistes de réflexion à partir desquelles il est possible de saisir s'il existe des comportements politiques propres à tel ou tel groupe social, de comprendre comment telle ou telle position sociale éclaire tel ou tel choix politique, de lire comment telle ou telle décision politique a pu influencer les configurations sociales. Bref, de mesurer l'ampleur des recompositions politiques et sociales engendrées par un régime se voulant tout à la fois l'héritier de la Révolution et le fondateur d'une France nouvelle non révolutionnaire, mais révolutionnée.

(9) Natalie Petiteau, Les Français et l'Empire, Paris, La Boutique de l'Histoire/Éditions universitaires d'Avignon, 2008, $278 \mathrm{p}$. 


\section{Trajectoires individuelles et collectives}

Le monde nobiliaire, en raison de l'abondance des sources qu'il a laissées derrière lui, a permis aux historiens de suivre les destins de nombreuses familles de part et d'autre de la Révolution et de l'Empire. Ces destins offrent des observatoires précieux. C'est en effet à propos des noblesses que la portée politique des réalisations sociales de l'Empire est la plus claire. Par ailleurs, c'est à l'endroit des noblesses anciennes que les volontés d'obtenir des ralliements ont été les plus fortes. Enfin, c'est par la noblesse impériale que Napoléon a tout particulièrement tenté de façonner la France post-révolutionnaire selon ses vues. Ses tentatives fondées initialement sur la Légion d'honneur n'ayant pas été satisfaisantes ${ }^{10}$, Napoléon a ainsi prolongé son entreprise de réorganisation de la hiérarchie sociale par la réorganisation des noblesses.

Or les destins nobiliaires illustrent en fait combien les persistances de l'Ancien Régime, malgré les années révolutionnaires et impériales, ont été fortes. Claude-Isabelle Brelot a ouvert la voie de leur étude : en mettant en œuvre les méthodes de la prosopographie à propos de la noblesse comtoise, elle a montré combien la noblesse a fait preuve d'adaptation et de résistance au point de réinventer l'idée de noblesse, et les nombreux ralliements à l'Empire n'y ont nullement fait obstacle. Les lendemains de la Révolution et de l'Empire laissent peu à peu se dégager, au sein de la nébuleuse nobiliaire, une élite nouvelle constituée des légitimistes fortunés ${ }^{11}$. Reste que les noblesses anciennes se sont pour partie acculturées aux données de la vie politique nouvelle, investissant charges locales, assemblées nationales et cabinets ministériels pour les ralliés, organisant des réseaux déterminants pour les forces contre-révolutionnaires dans le cas des opposants résolus. Plus précisément, la reconstitution de trajectoires d'émigrés prouve que certains ont pris leur distance avec les tenants d'une restauration pure et simple de l'Ancien Régime ${ }^{12}$. Et les revirements au sein du monde de l'émigration

(10) Natalie Petiteau, « De la Légion d'honneur à la noblesse d'Empire : la recomposition d'une hiérarchie sociale dans la France post-révolutionnaire », dans La Phalère. Revue européenne d'histoire des ordres et des décorations, $\mathrm{n}^{\circ}$ 1, 2000, p. 109-121; "Pourquoi Napoléon crée-t-il la Légion d'honneur? », dans Jean Tulard, François MonNier, Olivier ÉchapPÉ (dir.), La Légion d'honneur. Deux siècles d'histoire. Paris, Perrin, 2004, p. 35-48.

(11) Claude-Isabelle Brelot, La noblesse réinventée. Nobles de Franche-Comté de 1814 à 1870, Paris, Les Belles Lettres, 1992, p. 514 sq.

(12) Kirsty Carpenter, Philip Mansel (ed.), The French Emigrés in Europe and the Struggle against Revolution, 1789-1814, London, Macmillan Press, 1999, 236 p. 
ne sont pas rares ${ }^{13}$. Il existe même une noblesse de robe de fidélité royaliste, mais séduite par la rationalité et l'efficacité du système administratif de l'Empire, comme en témoignent Molé et Pasquier ${ }^{14}$.

Même si une minorité agissante a choisi, par l'émigration, le soutien actif à l'armée des Princes et aux réseaux royalistes ${ }^{15}$, dans nombre de villes de province, l'ancienne noblesse continue à tenir le haut du pavé, en Gironde comme dans la Somme par exemple. À Bordeaux en effet, les ralliements à Napoléon ne constituent pas la voie royale du retour au devant de la scène : quand ils existent, ils ne sont que de pure façade. Les nobles girondins préfèrent de beaucoup renouer rapidement avec leur cause traditionnelle et la fidélité aux Bourbons : ils se mobilisent donc dans l'ombre pour préparer la Restauration ${ }^{16}$. Dans la Somme, dès les lendemains de la Terreur, les nobles non émigrés s'affirment comme les personnages-clefs des villages picards. Mais le plus grand nombre des nobles de la Somme demeure réticent face à l'Empire, ce qui n'empêche pas les anciens privilégiés de tenir une place essentielle dans le nouveau groupe des notables ${ }^{17}$. Globalement la noblesse ancienne n'a pas été ruinée et elle a conservé une grande part de son influence. Le fait même que la noblesse d'Empire ait comporté une catégorie composée de nobles d'Ancien Régime indique la diversité des destins des membres de l'ancienne aristocratie. Certains itinéraires, au sein de cette noblesse refaite, montrent que le maintien de la position sociale, d'une noblesse à l'autre, était fait aussi de loyalisme à l'égard du pouvoir en place, de la Révolution à la Restauration en passant par l'Empire. Il en va ainsi, entre autres, de ce savant et grand commis de l'État qu'était Coquebert de Montbret ${ }^{18}$.

Pourtant, le jeu de recomposition des noblesses anciennes est perturbé par la création, en 1808 , de la noblesse d'Empire ${ }^{19}$. Napoléon veut tout à la fois, par l'attribution de titres d'Empire alors seuls reconnus, doter

(13) Karine Rance, «La Contre-Révolution à l'œuvre en Europe », dans Jean-Clément MARTIN (dir.), La Révolution à l'œuvre, op. cit., p. 190.

(14) Igor Moullier, "Bourgeoisie et bureaucratie au début du XIX siècle », dans JeanPierre Jessenne (dir.), Vers un ordre bourgeois?, op. cit., p. 237-253.

(15) Jean-Paul Bertaud, Les royalistes et Napoléon, Paris, Flammarion, 2009, 463 p.

(16) Michel Figeac, Destins de la noblesse bordelaise (1770-1830), Bordeaux, Fédération historique du Sud-Ouest, 1996, 989 p. en 2 volumes

(17) Jean-Marie Wiscart, La noblesse de la Somme au XIXe siècle, Amiens, Encrage, 1994, p. $57-92$.

(18) Isabelle Laboulais-Lesage, Lectures et pratiques de l'espace. L'itinéraire de Coquebert de Montbret, savant et grand commis de l'État (1755-1831), Paris, Champion, 1999, 753 p.

(19) Je me permets de renvoyer à la publication de ma thèse : Élite et mobilités : la noblesse d'Empire au XIX siècle (1808-1914), Paris, La Boutique de l'histoire, 1997, 714 p. 
son régime d'une noblesse qui le représente avec honneur dans les cours européennes, et d'une aristocratie qui lui permette non seulement de créer de l'émulation au sein de la société post-révolutionnaire, mais aussi de fusionner les élites anciennes et nouvelles. La noblesse impériale est donc présentée comme une noblesse de fonction à la portée de tous les mérites. Il y a là une réalisation sociale dont la visée politique est indéniable. Il s'agit en effet de constituer le socle de la quatrième dynastie, de façonner une élite dévouée à l'empereur et à ses descendants. « Masse de granit » destinée à consolider l'ensemble de la société française, la noblesse d'Empire est donc conçue aussi comme une aristocratie de vassaux dévoués à leur maître. La législation de 1808 le prouve en imposant aux titrés de prêter un serment les chargeant d'obligations auxquelles échappent les autres citoyens : chaque récipiendaire d'un titre impérial doit en effet jurer solennellement « d'être fidèle à l'empereur et à sa dynastie ».

Reste à savoir si cette ambition politique a trouvé satisfaction. Incontestablement l'espoir d'intégrer la noblesse impériale a pu contribuer à la soumission de beaucoup : un royaliste comme Meiffren Laugier de Chartrouse, maire d'Arles et grand propriétaire foncier, s'empresse de faire allégeance et de constituer un majorat pour obtenir un titre. Mais la noblesse d'Empire est plus encore constituée d'hommes qui, souvent dès 1789, ont fait le choix de la cause de la Révolution, du moins tant que celle-ci demeurait modérée. Bien des anoblis militaires ont été tout à la fois des enfants des Lumières et des patriotes convaincus d'agir pour la bonne cause lorsqu'ils triomphaient en Italie ou en Allemagne. Quant aux futurs anoblis ayant siégé dans les assemblées révolutionnaires, ils forment un groupe favorable à une Révolution bourgeoise et censitaire, mais inquiète devant l'égalitarisme et le dirigisme robespierristes.

Il n'est donc guère étonnant de constater que, en dépit de trajectoires sociales qui ont conduit les anoblis au sommet de la société française, leur adhésion au régime n'a pas été forcément à la hauteur des espoirs placés en eux par Napoléon. L'aristocratie impériale n'a nullement constitué le soutien unanime que la quatrième dynastie attendait. Ainsi, dès les préparatifs de la campagne de 1814, des réticences s'expriment au Sénat, travaillé par ceux de ses membres qui ont siégé dans des assemblées révolutionnaire ${ }^{20}$. Et finalement, sénateurs et maréchaux, fers de lance de la noblesse impériale, sont parmi les premiers artisans de la chute de l'Empire. Le changement de gouvernement place les anoblis face à une 
douloureuse alternative : sauver leur honneur en demeurant fidèles à la dynastie à laquelle ils doivent leur ascension ou le préserver en obéissant au nouveau souverain incarnant désormais le pouvoir légal. La majorité de ceux qui jouent un rôle de premier plan choisit la seconde solution. La propension à une docilité au pouvoir établi a pesé lourd dans bon nombre de ralliements d'officiers qui, par ailleurs, étaient préoccupés, comme les sénateurs, de sauver non pas seulement leur honneur, mais aussi leur position sociale. Au final, des ordonnances de juillet 1815 organisant l'épuration, aux tentatives de Louis-Napoléon à Strasbourg et à Boulogne sous la Monarchie de Juillet, les lendemains de l'Empire révèlent qu'il n'a existé qu'un noyau de bonapartistes activistes au sein d'une noblesse impériale qui n'est guère au rendez-vous autour des héritiers de l'empereur ${ }^{21}$.

Si l'on passe de la prosopographie à la biographie, les brillants renouvellements de ce genre livrent également des itinéraires tout à fait significatifs. Ainsi Merlin de Douai est-il l'archétype des avocats engagés avec conviction dans la Révolution : convaincu du bien-fondé du renversement de l'absolutisme, il est hostile à la voie jacobine de la Révolution et se rallie aux thermidoriens puis à l'Empire. Son cheminement révèle comment des révolutionnaires convaincus ont pu nourrir le goût du pouvoir et des honneurs au point d'intégrer la nouvelle noblesse ${ }^{22}$. D'autres travaux récents détaillent les engagements révolutionnaires de membres de l'ancienne noblesse, tel Félix Lepeletier de Saint Fargeau. Proche de Robespierre, mais protégé aussi de Tallien et de Barras, opposant notoire à Brumaire, il n'en devient pas moins maire de sa commune normande en $1811^{23}$. Quant aux officiers de l'Empire issus de catégories modestes, ils forment après la dissolution de l'armée, de par leur position dans la hiérarchie militaire, une très modeste bourgeoisie auréolée du prestige de ses victoires, jouissant de médiocres pensions mais vouée, pour maintenir son rang, à ne pas dépendre d'une activité salariée. Reste que, même à l'issue de telles trajectoires, les fidélités à la cause napoléonienne ne sont nullement systématiques ${ }^{24}$.

(21) Natalie Petiteau, « Identité sociale et engagement politique : les anoblis de Napoléon I $^{\text {er }}$ face au bonapartisme (1814-1870) », Revue européenne d'histoire, 1996, n 2, p. 161-170.

(22) Hervé Leuwers, Un juriste en politique : Merlin de Douai (1754-1838), Arras, Artois Presses Université, 1996, 378 p.

(23) Laurence Constant, Félix Lepeletier de Saint Fargeau. Un itinéraire de la Révolution à la Monarchie de Juillet, Paris, Découvrir, 1995, 199 p.

(24) Natalie Petiteau, Lendemains d'Empire. Les soldats de Napoléon dans la France du XIX siècle, Paris, La Boutique de l'Histoire, 2003, 400 p.; Stéphane CALvet, Destins de braves. Les officiers charentais de Napoléon au XIX ${ }^{e}$ siècle, Paris, La Boutique de l'histoire/Éditions universitaires d'Avignon, à paraître en 2010. 
Ces biographies sociales sont à replacer dans le cadre plus général des destins de la bourgeoisie au tournant des XVIII ${ }^{\mathrm{e}}$ et XIX ${ }^{\mathrm{e}}$ siècles. On doit ici mentionner pour mémoire le débat sur la bourgeoisie à cette période, car il est revenu à l'ordre du jour sous l'influence des travaux de Sarah Maza qui contestent l'existence même d'une bourgeoisie à la fin du XVIII ${ }^{\mathrm{e}}$ siècle. Ils soulignent que les élites de l'Empire, qui ne constitueraient pas une vraie bourgeoisie, se distinguent par leur engagement commun au service de l'État, mais ils ne voient ni les finalités politiques de la création de la noblesse impériale, ni la forte part de la propriété foncière dans les fondements de l'affirmation des élites nouvelles ${ }^{25}$.Quant aux travaux d'Henry Heller, eux aussi fondés sur un travail de bibliographie et non pas sur le dépouillement de sources, ils ne font que reprendre le constat, somme toute classique, selon lequel hors de Paris, la bourgeoisie domine les instances politiques locales et forme avec l'ancienne noblesse le monde de la notabilité ${ }^{26}$.

Puisque l'on se demande aujourd'hui encore si la Révolution française a été une révolution bourgeoise ${ }^{27}$, il faut aussi savoir si l'Empire a donné satisfaction à la bourgeoisie et lui a permis de maintenir son emprise politique. Pour ce qui est des instances administratives locales, on peut finalement repérer des continuités de part et d'autre de 1799. Des biographies ont montré la tendance à une double continuité patrimoniale et politique, comme par exemple chez Boissy d'Anglas ${ }^{28}$. De telles continuités mériteraient néanmoins des études plus systématiques, et l'ont peut regretter que les travaux de Catherine Kawa n'aillent pas au-delà de 1800 ; ils permettraient de mieux connaître une petite bourgeoisie liée à l'appareil administratif ${ }^{29}$. Ceux d'Igor Moullier viennent en revanche fournir d'importants éclairages sur un autre échelon des serviteurs de l'État : ils soulignent entre autres que les ministres de l'Intérieur de Napoléon, à l'exception de Champagny, membre de l'ancienne noblesse, viennent tous de la bourgeoisie, rurale pour Laplace et Chaptal, négociante pour

(25) Sarah Maza, The Myth of the French Bourgeoisie. An Essay on the Social Imaginary, 1750-1850, Harvard University Press, 2003, 255 p.

(26) Henry Heller, The Bourgeois Revolution in France, 1789-1815, New York-Oxford, Berghan Books, 2006, 172 p.

(27) Martine Lapied, Christine Peyrard (dir.), La Révolution française au carrefour des recherches, op. cit., p. 72.

(28) Christine Le Bozec, Boissy d'Anglas, un grand notable libéral, Privas, Fédération des Euvres Laïques de l'Ardèche, 1995, 503 p.

(29) Catherine Kawa, Les Ronds-de-cuir en Révolution : les employés du Ministère de l'Intérieur sous la Première République (1792-1800), Paris, Editions du Comité des travaux historiques et scientifiques, 1996, $583 \mathrm{p}$. 
Cretet, juridique pour Montalivet ${ }^{30}$. Pour ce qui est de l'ensemble des collaborateurs de Napoléon, Isser Woloch a amplement montré comment ils sont pour la plupart des hommes de la Révolution. Sans cependant faire le point sur les appartenances sociales des uns et des autres, il met en évidence la véritable méritocratie que constituent les sénateurs, en soulignant la continuité du service de l'État qui caractérise le corps préfectoral ${ }^{31}$.

On peut ici regretter que la série des Grands notables du Premier Empire ne soit pas achevée ${ }^{32}$ et qu'elle n'ait pas encore donné lieu à une synthèse véritable sur les trajectoires ainsi reconstituées. L'ensemble des notices témoigne d'ores et déjà de l'adhésion théorique d'un monde de la notabilité ne comportant que peu de noms tout à fait nouveaux, mêlant fonctionnaires dévoués au nouveau régime, rentiers du sol et ancienne noblesse. Dans la Vienne par exemple ${ }^{33}$, la notabilité impériale est bien davantage proche de celle de la Restauration que de celle de la Révolution, elle intègre du reste fort discrètement les rescapés politiques de l'ère révolutionnaire. Mais il convient d'observer de plus près les réactions de tel ou tel notable. On voit ainsi que Benoît Lacombe, à Gaillac, vit au rythme des annonces de guerre et de paix, des nouvelles relatives aux alliances qui sont entre les mains des politiques. En 1803-1804, il devient un ardent propagandiste de l'idée de nation et se révèle en quelque sorte fasciné par l'aptitude de Bonaparte à l'incarner. Mais son enthousiasme retombe dès 1807 et plus aucune référence à Napoléon n'apparaît dans ses lettres après 1809. La correspondance de Lacombe atteste que l'attente de la paix a gommé toute la confiance qu'il avait placée dans le nouveau régime ${ }^{34}$. Dans la Loire, les négociants stéphanois se désintéressent en fait de la nature du régime. Quant à ceux qui, parmi les notables, occupent des fonctions administratives, ils se montrent soucieux de servir le pays bien plus que le régime, ce qui est un aspect essentiel de l'histoire socio-politique pour l'ensemble de la période contemporaine ${ }^{35}$. L'attitude même de cer-

(30) Igor Moullier, op. cit.

(31) Isser Woloch, Napoleon and his Collaborators. The Making of a Dictatorship, New York-London, Norton and Company, 2001, 281 p.

(32) Louis Bergeron, Guy Chaussinand-Nogaret (dir.), Grands notables du Premier Empire. Notices de biographies sociales, Paris, CNRS, 28 volumes parus depuis 1978.

(33) Guillaume LÉVÊQUE, Emmanuel Dion, Sébastien JAHAN, Grands notables du Premier Empire. Vienne, Paris, CNRS éditions, 2000, 278 p.

(34) Joël CORNETTE, Un révolutionnaire ordinaire : Benoît Lacombe, négociant (1759-1819), Paris, Champ Vallon, 1986, p. 264-270.

(35) Voir par exemple Frédéric Monier, La politique des plaintes. Clientélisme et demandes sociales dans le Vaucluse d'Édouard Daladier (1890-1940), Paris, La Boutique de 1'histoire, 2007, $411 \mathrm{p}$. 
tains cadres ou de notables d'envergure strictement locale a pu contribuer à miner le régime de l'intérieur ${ }^{36}$. À Paris, les quinze négociants de la Chambre de commerce n'hésitent pas à affirmer une opposition de principe aux guerres impériales nuisibles au commerce et à se dresser contre le système des licences ${ }^{37}$. Mais globalement les négociants apprécient de se voir reconnus comme hommes éclairés et loyaux citoyens, ce qui favorise là aussi bien des ralliements, y compris au sein des collèges électoraux ${ }^{38}$. Les drapiers de Sedan et d'Elbeuf, ou les négociants de Lille prouvent que la bourgeoisie qui a exprimé nettement ses sympathies pour la Révolution en 1789 jouit des honneurs, de la prospérité et des charges politiques sous l'Empire ${ }^{39}$.

Dans le monde des campagnes, le poids des grands propriétaires forains dans la direction des municipalités du Toulousain, sous l'Empire, illustre l'émergence d'élites nouvelles qui ont réussi à surmonter les aléas de la vie politique révolutionnaire ${ }^{40}$. Ailleurs, la bourgeoisie rurale en place avant 1789 fait preuve d'une réelle adaptation dont témoigne son accès aux fonctions locales ${ }^{41}$. L'étude des notables des Mauges a montré comment la refonte des organisations étatiques, du Directoire à la Restauration, engendre des conditions nouvelles d'émergence de notabilités, d'autant mieux que l'État, pour la reconstruction, a besoin d'interlocuteurs locaux. Peu à peu, une notabilité politique émerge, désormais composée presque exclusivement des élites agricoles et foncières. Par ailleurs, le pouvoir central trouve d'autres interlocuteurs au sein de l'ancienne noblesse terrienne, élite jusqu'alors marginalisée dans la vie politique locale, can-

(36) Pascal Chambon, La Loire et l'Aigle. Les Foréziens face à l'État napoléonien, Publications de l'Université de Saint-Étienne, 2005, 574 p.

(37) Claire LemERCIER, Un si discret pouvoir. Aux origines de la Chambre de commerce de Paris, 1803-1853, Paris, La Découverte, 2003, p. 146-147.

(38) Gérard GAYOT, «Quand les chefs de manufacture et les gens de travail retrouvèrent leur place naturelle dans la société, après Brumaire an VIII », dans Jean-Pierre JESSENNE (dir.), Du Directoire au Consulat. Tome 3 : Brumaire dans l'histoire du lien politique et de l'État-nation, LilleRouen, Centre de recherche sur l'histoire du Nord-Ouest et Groupe de recherches historiques, 2001, p. 239.

(39) Gérard GAYot, Les draps de Sedan, 1646-1870, Paris, Éditions de l'EHESS et Terres ardennaises, 1998, 579 p. ; Alain BECCHIA, La draperie d'Elbeuf des origines à 1870, Rouen, Publications de l'Université de Rouen, 2000, 869 p.; Jean-Pierre HiRsch, Les deux rêves du commerce. Entreprises et institutions dans la région lilloise 1780-1860, Paris, éditions de l'EHESS, 1991, 534 p.

(40) Georges Fournier, Démocratie et vie municipale en Languedoc du milieu du XVIII siècle au début du XIX siècle, Toulouse, Association des Amis des Archives de la HauteGaronne, 1994, tome 2, p. 312.

(41) Jean-Pierre JESSENNE, « Usages, équivoques et pertinence de "bourgeoisie rurale" », dans Jean-Pierre JESSENNE (dir.), Vers un ordre bourgeois ?, op. cit., p. 140. 
tonale ou départementale. On voit ainsi comment le conflit vendéen a légitimé la place de la noblesse, comment il a créé des liens entre châtelains et paysans. La reconstitution des destins met finalement en évidence comment les ruraux en viennent à participer à la vie politique, mais aussi comment la guerre, contingence politique elle aussi, est productrice de cadres sociaux, comment les réseaux se tissent et fonctionnent, comment s'élaborent des espaces spécifiques du politique et du social ${ }^{42}$.

\section{Espaces spécifiques}

Au-delà de ce que révèlent les trajectoires, l'espace des élites permet d'observer, à un moment précis, la diversité des positionnements politiques dans des situations sociales similaires. Ainsi, en Haute-Garonne, le conseil général a offert aux élites sociales qui le composent un espace de critiques. En 1807, les conseillers s'en prennent vigoureusement aux contributions indirectes et à l'attitude des fonctionnaires d'autorité qui ne consultent pas les hommes d'expérience, mais qui décident tout depuis leurs bureaux en traitant les administrés avec désinvolture. Or, si cette attitude critique a suscité la colère de Napoléon, le Ministère le dissuade de publier une circulaire de rappel afin de tenter d'ensevelir une telle résistance dans l'oubli ${ }^{43}$. C'est dire que l'on sait que la contagion serait aisée... Le royalisme peut-être plus prononcé de certains conseillers toulousains n'entre ici pas seul en compte. Dans cette attitude des conseils locaux se lit en fait celle des hommes qui encadrent la vie commerçante et manufacturière du pays. $\mathrm{Si}$, à l'origine, les négociants français apprécient la sécurité et la stabilité politique et sociale que le Consulat et l'Empire leur assurent, si le régime leur ouvre les portes des structures politiques et représentatives et leur garantit la reconnaissance sociale à laquelle ils aspirent, la participation politique du négoce a finalement tiédi avec le temps. On note certes un nombre assez élevé de négociants-édiles, mais leur participation n'est pas toujours empressée. La défaite en Russie fait perdre les dernières sympathies et pousse le monde du négoce à l'absentéisme, à l'absence d'empressement face aux fonctions administratives, à l'indifférence lors des élections ou lors des manifestations officielles,

(42) Anne Rolland-Boulestreau, Les notables des Mauges. Communautés rurales et Révolution (1750-1830), Rennes, Presses Universitaires de Rennes, 2004, 401 p.

(43) Germain SiCARD, « Les opinions politiques des notables toulousains durant l'Empire et la Restauration d'après les délibérations du conseil général de Haute-Garonne », dans Réflexions idéologiques sur l'État. Aspects de la pensée politique méditerranéenne, Presses de l'Université d'AixMarseille, 1987, p. 325-334. 
et finalement à une opposition telle que les villes maritimes montrent en général un grand enthousiasme au retour des Bourbons ${ }^{44}$.

À l'issue de la Révolution, en raison des épurations successives, l'armée est devenue un bastion de républicanisme, et c'est de cette armée là dont hérite Bonaparte qui a lui-même fait figure de protecteur du régime républicain. Pourtant il éloigne bientôt à Saint-Domingue certains officiers aux opinions républicaines les plus vives, d'autres, après l'affaire Pichegru et le complot Cadoudal, sont résolument écartés, tels Moreau ou Lecourbe. Parce qu'il lui doit sa fondation, le régime place l'armée au cœur de la France impériale. Force de répression à l'intérieur et outil de conquête à l'extérieur, l'armée façonne la société nouvelle ${ }^{45}$. Elle est elle aussi un espace social dans lequel se lit la complexité des attitudes politiques des Français. Toutes les tendances politiques nées de la Révolution coexistent parmi les généraux, Augereau, Bernadotte ou Bessières peuvent être classés au rang des jacobins déclarés, mais il existe à l'inverse bien des généraux regardés comme des $«$ amis du roi $»^{46}$. Au total, le ralliement des officiers est trompeur, il masque de nombreuses consciences restées démocrates ou radicales ${ }^{47}$. Des velléités d'opposition apparaissent dans les périphéries du Grand Empire, souvent appuyées sur des réseaux clandestins, tel celui des philadelphes. Elles sont pour certaines révélées par le complot de Malet. Et si à l'inverse une forte majorité exprime son attachement à Napoléon, il n'est pas forcément synonyme d'adhésion au régime, encore moins d'un bonapartisme qui n'existe pas encore avant $1815^{48}$. Les lendemains de l'Empire prouvent en revanche comment nombre d'officiers - bourgeois ou membres des noblesses, officiers souvent sortis du rang - se réunissent dans les rangs des mouvements libéraux : patriotes pas forcément attachés à la cause de Napoléon II, ils ont en commun d'être des déçus de la Restauration ${ }^{49}$.

L'étude de l'espace social militaire rappelle par ailleurs qu'il y a eu des centaines de milliers de réfractaires et insoumis pour témoigner

(44) Silvia Marzagalli, Les boulevards de la fraude. Le négoce maritime et le blocus continental, 1806-1813, Lille, Presses Universitaires du Septentrion, 1999, 396 p.

(45) Jean-Paul Bertaud, Quand les enfants parlaient de gloire. L'armée au cour de la France de Napoléon, Paris, Aubier, 2006, 460 p.

(46) Idem, p. 65.

(47) Bernard GAINot, «L'opposition militaire autour des sociétés secrètes dans l'armée », $A H R F, \mathrm{n}^{\circ}$ spécial Les héritages républicains sous le Consulat et l'Empire, 2006, $\mathrm{n}^{\circ}$ 4, p. 45-58.

(48) Walter BRUYĖRE-Ostells, «Les officiers républicains sous l'Empire : entre tradition républicaine, ralliement et tournant libéral », AHRF, $\mathrm{n}^{\circ}$ spécial Les héritages républicains sous le Consulat et l'Empire, 2006, n ${ }^{\circ}$ 4, p. 31-44.

(49) Walter Bruyère-Ostells, La Grande Armée de la liberté, Paris, Tallandier, 2009, 335 p. 
de la façon dont le refus du régime a pu s'ancrer dans les campagnes. L'attitude des populations face à la conscription a fait l'objet de quelques belles approches régionales et de plusieurs synthèses ${ }^{50}$. Jusqu'en 1806, de graves incidents éclatent encore, en dépit de la relative faiblesse des levées d'hommes des premiers temps. C'est ensuite l'apaisement, en raison de l'efficacité de la répression, des formes plus subtiles d'insoumission, mais aussi de l'acceptation du service de la patrie. La propagande a même pu produire des effets passagers ${ }^{51}$, tandis que les Français ont été finalement avides de gloire. En revanche, avec les très importantes levées de 1813, ce sont de véritables émeutes qui éclatent contre la conscription. L'alourdissement des levées d'hommes a alors mis à mal la «naturalisation» de la conscription $^{52}$. Toutefois certaines analyses tendent à montrer qu'à partir de 1810, la réduction des cas de désertion est très nette. Ensuite, à part quelques incidents isolés, les recrutements de 1812 et 1813 se seraient effectués sans difficulté ${ }^{53}$. Reste que la fraude et l'insoumission ont persisté tout au long de l'Empire, facilitées par l'aide familiale plus encore que communautaire ${ }^{54}$. Elle a pu conduire à de véritables révoltes, ainsi la commune de Collioure devient ingouvernable à partir de $1813^{55}$.

L'attitude politique à l'égard du régime n'explique cependant pas seule les réactions à la conscription : les habitudes de résistances à l'État centralisateur ont également souvent comptés ${ }^{56}$, les réseaux de migrations

(50) Louis BERgÈs, Résister à la conscription, 1798-1814: le cas des départements aquitains, Paris, CTHS, 2002, 598 p. ; Annie CRÉPIN, La conscription en débats ou le triple apprentissage de la nation, de la citoyenneté, de la République (1798-1889), Arras, Artois Presses Université, 1998, 253 p.; Alan Forrest, Déserteurs et insoumis sous la Révolution et l'Empire, Paris, Perrin, 1988, 326 p.; Alan Forrest, Napoleon's Men, The Soldiers of the Revolution and Empire, Londres-New York, Hambledon and London, 2002, 248 p.; Frédéric RoussEAu, Service militaire au XIX siècle : de la résistance à l'obéissance. Un siècle d'apprentissage de la patrie dans le département de l'Hérault, Montpellier, UMR 5609-ESID, 1998, 224 p.

(51) Annie CRÉPIN, Défendre la France. Les Français, la guerre et le service militaire de la guerre de Sept ans à Verdun, Rennes, Presses Universitaires de Rennes, 2005, p. 167.

(52) Annie CRÉPIN, La conscription en débats, op. cit., p. 114-115.

(53) Alan ForRest, «La formation des attitudes villageoises envers le service militaire: 1792-1814 », dans Paul Viallaneix, Jean Ehrard (dir.), La bataille, l'armée, la gloire, 1845-1871, tome 1, Clermont-Ferrand, Publications de la Faculté des Lettres et Sciences Humaines de ClermontFerrand, 1985, p. 171-182.

(54) Alan Forrest, Déserteurs et insoumis, op. cit.; Kôbô SeIgan, La conscription dans le département de la Seine Inférieure (an VI-1815), thèse pour le doctorat sous la direction de Jean-Paul Bertaud, Université de Paris I, 1998, 548 p.

(55) Les pratiques politiques en province à l'épreuve de la Révolution française, Montpellier, Publications du Centre d'histoire contemporaine du Languedoc méditerranéen et du Roussillon, 1988 , p. 244.

(56) Annie CRÉPIN, La conscription en débats, op. cit., p. 115. 
de travail aussi ${ }^{57}$, tandis que des patriotismes locaux ont pu rendre sourd aux appels de la grande patrie ${ }^{58}$. Bien des attitudes s'éclairent de l'analyse des mentalités et des structures économiques et sociales : dans le cas des départements aquitains par exemple, Louis Bergès a bien montré l'impact de la crainte du déracinement et l'aversion pour l'existence incertaine. La distance envers l'État-nation, le défaut de patriotisme ou plutôt l'attachement primordial à la petite patrie ont également été déterminants ${ }^{59}$. Les freins à la conscription peuvent résider plus encore dans les structures économiques et sociales. Car la conscription peut mettre à mal le fonctionnement des exploitations familiales, qu'il s'agisse de petites propriétés, de fermes ou de métairies ${ }^{60}:$ dans les départements aquitains aussi, on repère cette anxiété devant la rupture de fragiles équilibres économiques. Les motivations du refus existent partout et tout au long de la période, ne serait-ce qu'en raison de l'impact des récits des soldats qui reviennent du front.

L'armée est décidément un excellent observatoire de la façon dont s'entremêlent les données du politique et du social pour dessiner les attitudes des populations. Du reste, en prenant pour observatoire de l'histoire socio-politique les hommes qui ont en commun d'avoir fait partie, pour un temps plus ou moins long, de l'armée napoléonienne, on mesure la diversité des attitudes et des destinées, notamment pour cette minorité emblématique des officiers de couleur ${ }^{61}$. Pour nombre de Français modestes, le passage sous les drapeaux a été certes un temps de politisation, mais qui n'a pas conduit forcément au bonapartisme, il s'en faut de beaucoup. Pour une minorité cependant, pour qui l'armée a pu être un moyen, même modeste, de promotion sociale, la politisation a pu conduire à soutenir résolument, même après 1815, la cause des Bonaparte, plus souvent peutêtre même que dans le cas des anoblis ${ }^{62}$.

Aurélien Lignereux a pour sa part attiré l'attention sur les espaces en rébellion : l'étude des rébellions à la gendarmerie a révélé comment, dans ces actes, s'expriment des refus du régime. Il en va ainsi, par exemple, lorsque des villageois de Monnières (Loire-Inférieure) se mobilisent, en

(57) Abel Chatelain, « Résistance à la conscription et migrations temporaires sous le Premier Empire », AHRF, 1972, p. 606-625.

(58) Annie CRÉPIn, Défendre la France, op. cit., p. 167.

(59) Louis BERGÈs, Résister à la conscription, op. cit.

(60) Annie CréPIN, La conscription en débats, op. cit., p. 168.

(61) Bernard Gainot, Les officiers de couleur dans les armées de la République et de l'Empire (1792-1815) - De l'esclavage à la condition militaire dans les Antilles françaises, Paris, Karthala, 2007, 232 p.

(62) Natalie Petiteau, Lendemains d'Empire, op. cit. 
juillet 1803, pour défendre quatre jeunes gens arrêtés pour avoir porté la cocarde blanche. On se trouve certes ici dans les provinces de l'Ouest où le sens politique des rébellions est plus affirmé qu'ailleurs. Reste que l'on repère de nombreuses attaques menées en vue de libérer des insoumis, attaques qui ne cessent de se multiplier après 1813. Mais l'insoumission ne saurait être confondue avec la rébellion : « 310 rébellions, soit le tiers de celles de la période 1800 -octobre 1813, éclatent pour d'autres motifs, témoignant de la persistance d'un fond rébellionnaire plus large, hérité de l'Ancien Régime et ravivé par l'augmentation du nombre de fonctionnaires et par la fixation des lois et des règlements sous Napoléon $»^{63}$.

Par ailleurs, une enquête menée sur les procès politiques sous Napoléon révèle que le chef d'accusation le plus commun est celui d'avoir tenu des propos séditieux. Les crimes de parole représentent un tiers des affaires politiques : la plupart des personnes prévenues à ce titre sont soupçonnées de sympathies royalistes et sont souvent accusées d'avoir crié «Vive le roi » ou «Vive mon Louis XVIII », «Vive le roy, vive la reine, vive le comte d'Artois $»^{64}$. En fait, les attitudes à l'égard de la personne qui incarne la nation demeurent proches de celles qui existaient avant $1789^{65}$. On exprime son rejet de la situation présente en fustigeant le chef de l'État et parfois même son épouse, preuve de la prégnance des cadres de pensée hérités des temps monarchiques. Ainsi, en septembre 1801, une affiche placardée sur un arbre du boulevard des Capucines, à Paris, comporte des propos injurieux à la fois contre le premier consul et contre Madame Bonaparte ${ }^{66}$. Avec 1'Empire, se multiplient les traces d'injures envers Napoléon. Peu après la proclamation officielle du nouveau régime, on entend, dans les réunions publiques, beaucoup de réflexions malveillantes sur la titulature « Napoléon empereur des Français » qui ne serait déjà plus suivie de la mention " par les constitutions de la République $»^{67}$. Pour une partie des Français, dont la proportion demeure impossible à estimer, la Révolution n'est pas oubliée, et son achèvement en un retour à une monarchie n'est pas accepté68.

(63) Aurélien Lignereux, La France rébellionnaire. Les résistances à la gendarmerie (18001859), Rennes, Presses Universitaires de Rennes, 2008, p. 31-33.

(64) Robert Allen, Les tribunaux criminels sous la Révolution et l'Empire, 1792-1811, Rennes, PUR, 2005, p. 220.

(65) Arlette FARGe, Dire et mal dire, op. cit., p. 197-286.

(66) $\mathrm{AN}, \mathrm{F}^{7} 3053$.

(67) $\mathrm{AN}, \mathrm{F}^{7} 3832$.

(68) Pour de plus amples précisions, je me permets de renvoyer à mon dernier ouvrage : Les Français et l'Empire, op. cit. 
Cependant, au fur et à mesure que le régime s'installe dans la durée, les propos séditieux perdent leurs références explicites aux Bourbons ou à la République. Ils se font plus rares aussi, du moins à en croire la chronologie que les mentions dans les bulletins de police permettent d'esquis$\operatorname{ser}^{69}$. Mais ils contiennent avant tout des mots hostiles au souverain, des injures : l'espace de la rébellion demeure, et c'est là que l'on peut entendre les positionnements politiques des anonymes, d'hommes et de femmes placés aux rangs les plus modestes de la hiérarchie sociale, du monde des artisans et ouvriers à celui même des marginaux. Dans leur refus du régime, les Français osent dire, pour certains, une haine véritable à l'égard de celui qui en est le maître. Ils intériorisent d'autant mieux les cadres politiques dans lesquels ils vivent que ceux-ci sont apparemment identiques à ceux de l'Ancien Régime. Leur expression politique tient avant tout en une détestation de celui qui incarne la nation, mais sans doute parce que la Révolution a fait son œuvre, osent-ils plus qu'avant injurier un souverain qui, en dépit de la cérémonie du 2 décembre 1804, semble, aux yeux de ces anonymes, dépourvu de toute sacralité. Par-delà les apparences, donc, d'une vie politique se déroulant encore dans les cadres intériorisés de l'absolutisme, se lit ainsi sans doute l'un des changements majeurs induits par la décennie révolutionnaire.

Pourtant, les lendemains de la Révolution sont marqués aussi par la réaction, dont Jean-Clément Martin a démontré qu'elle est particulièrement lisible dans l'espace social féminin, même si l'Empire n'a pas négligé, bien sûr, la législation sur le divorce. Reste que la société nouvelle est aussi organisée autour du principe de virilité, y compris dans une vie de cour où les évolutions de la mode traduisent les mutations du statut des femmes de la Révolution à l'Empire ${ }^{70}$. Au-delà des grands bals curiaux, l'espace des fêtes et des spectacles est également un lieu qui permet à l'historien de saisir comment le régime s'emploie à conquérir l'adhésion des populations et comment celles-ci répondent aux entreprises de propagande. Les directeurs de théâtre mettent en scène les batailles, moyennant quoi l'imaginaire public se militarise. Sur les places publiques, les mon-

(69) En suivant le découpage chronologique des volumes publiant ces bulletins on relève :

- 73 propos séditieux du 12 juillet 1804 au 11 juillet 1805 ,

- 67 propos séditieux du 12 juillet 1805 au 30 septembre 1806 ,

- 62 propos séditieux du $1^{\text {er }}$ octobre 1806 au 31 décembre 1807 ,

- 43 propos séditieux du $1^{\text {er }}$ janvier 1808 au 31 mars 1809 ,

- 42 propos séditieux du $1^{\mathrm{er}}$ avril 1809 au 30 mai 1810.

(70) Jean-Clément Martin, La révolte brisée. Femmes dans la Révolution française et l'Empire, Paris, Armand Colin, 2008, p. 175-240. 
treurs d'images déroulent les dessins des combats, partout, des poètes improvisés célèbrent les vertus guerrières de l'armée et les conquêtes de l'empereur ${ }^{71}$. Et tandis que les fêtes organisées lors des grands événements ou lors des grandes victoires sont orchestrées par les édiles municipaux de façon à ce que les louanges de l'empereur soient partout chantées avec la même ferveur au même moment, l'espace festif villageois n'en bruisse pas moins, par ailleurs, d'expressions d'oppositions spontanées de la part de ruraux finalement plus politisés qu'on ne le croit souvent ${ }^{72}$. Les récents travaux de Cyril Triolaire ont montré comment représentations théâtrales et festivités publiques jettent une lumière nouvelle sur la profondeur de la conscience politique parmi les gens ordinaires. Dans les départements du Massif Central comme ailleurs, célébrer n'est certes pas adhérer, mais fêtes et représentations théâtrales révèlent comment de nombreux Français sont mus par une même admiration du héros, grand homme et empereur de guerre, dont la perception apparaît particulièrement bien en des circonstances précises ${ }^{73}$.

\section{Moments clefs}

Les contemporains de l'année 1804 disent combien, dès ses origines, le régime impérial suscite de profondes réserves. En juillet 1804, Stendhal observe la tiédeur des réactions, alors que l'Empire est proclamé depuis peu et que Napoléon se rend en grandes pompes aux Invalides pour une très solennelle distribution de croix de la Légion d'honneur ${ }^{74}$. Or, la tiédeur des adhésions frappe encore les témoins des cérémonies de décembre. Le colonel Noël, qui assiste aux fastes du couronnement, note, à propos des réactions sur le passage du cortège, que les enthousiasmes réels sont rares ${ }^{75}$. Tandis que la Révolution avait inauguré des formes diverses de respect de la souveraineté populaire, l'essence même du régime impérial manifeste qu'il n'y a de nouveau plus, une fois le

(71) Jean-Paul Bertaud, Quand les enfants parlaient de gloire, op. cit., p. 249 sq.

(72) Claude Allègre, De la fête officielle à l'action politique autonome: l'exemple du Vaucluse sous le Consulat et le Premier Empire (1799-1815), mémoire de maîtrise, Université d'Avignon, 2001, $163 \mathrm{p}$.

(73) Cyril Triolaire, Fêtes officielles, théâtres et spectacles de curiosités dans le $11^{e}$ arrondissement théâtral impérial pendant le Consulat et l'Empire. Pouvoir, artistes et mises en scène, thèse pour le doctorat sous la direction de Philippe Bourdin, Université Blaise Pascal-Clermont II, 2008, p. 1262-1265 notamment.

(74) Stendhal, Journal dans Euvres intimes, Paris, Gallimard, Pléiade, 1955, p. 483.

(75) Colonel NoËL, Souvenir militaire d'un officier du Premier Empire (1795-1832), Paris, À la Librairie des Deux Empires, 1999, p. 24. 
plébiscite effectué, de réelle prise en compte de l'opinion. Pourtant le peuple ne semble pas renoncer docilement à toute expression d'un avis sur la façon dont sont conduites les affaires de la cité. Proclamé souverain en 1789 , il a été invité à remettre sa souveraineté entre les mains d'un homme qui prétend être son seul véritable représentant, qui prétend clore la Révolution en opérant une synthèse entre l'ordre et la démocratie. Mais privée des occasions de s'exprimer par le vote, placée sous un régime policier qui doit empêcher toute manifestation d'hostilité ouverte, la nation persiste à exister en tant que corps politique.

Les sources provenant du ministère de l'Intérieur attestent que s'expriment des hommes et des femmes inscrits dans un univers culturel qui n'est pas toujours celui de l'absolue maîtrise de l'écriture. Nombre de leurs adresses sont ostensiblement datées de l'an I du règne de Napoléon, et toutes placent l'empereur au-delà même du rang de héros. Nombre d'anonymes osent prendre une plume fort maladroite pour dire un enthousiasme qui relève bien peu de réflexes politiques des temps révolutionnaires, mais davantage des cadres de pensée forgés du temps de la royauté. Le « deuxième sexe », même dans ses strates les plus modestes, n'est d'ailleurs pas absent de l'espace politique, en ces temps où il existe pourtant une forte volonté de contrôle de l'empire des femmes ${ }^{76}$. C'est en tout cas par de telles attitudes que s'est en partie forgée précocement la légende napoléonienne, c'est au travers de tels comportements que s'exprime une partie de la France des humbles, prompts à vénérer un souverain en qui l'on voit encore un don de Dieu. Souvent spontanées et maladroites, ces adresses viennent en complément de toutes celles qui sont publiées dans la presse et de tous les discours prononcés dans les Chambres. Par leur spontanéité, elles élargissent l'espace politique jusqu'à des marges impensées par les gouvernants d'alors. L'historiographie a toujours oublié que l'Empire a aussi puisé sa légitimité à cette source. Les enthousiasmes de 1815 et le bonapartisme populaire du premier $\mathrm{XIX}^{\mathrm{e}}$ siècle trouvent leurs racines dans cette ferveur qui affleure dès 1804. Napoléon est très vite accepté comme fondateur d'une dynastie : les réactions évoquées ci-dessus indiquent combien les craintes pour sa vie seraient atténuées s'il avait un descendant. Il semble en fait qu'il soit d'autant mieux apprécié qu'il s'est inscrit dans des cadres de pensée familiers pour les Français d'alors. On sait que le respect pour la personne du roi était encore largement répandu en 1789; le fait même que l'homme 
qui s'est montré apte à achever la Révolution se soit fait monarque a comme soulagé une partie de l'opinion qui peut de nouveau lire la vie politique au travers d'une grille d'interprétation familière et faisant facilement sens.

Pourtant, en 1812, le tableau politique est déjà bien différent : en raison de la crise de l'économie, l'Empire a cessé d'être assimilé à la prospérité, à l'heure où les défaites ne permettent plus d'enrichir le Trésor public par les prélèvements sur les pays vaincus. Si bien que, en différents lieux, les Français qui souffrent de la cherté des grains expriment sur des placards apposés en place publique leur exaspération contre la hausse des prix et contre les ventes de blés hors des lieux de leur production $^{77}$. Certes, les accusations se portent avant tout contre les autorités locales et non contre l'empereur. En dépit du fait que le gouvernement intervient par des prêts et des commandes à de grandes entreprises, par une politique de grands travaux et par l'importation de grains étrangers, il est jugé comme seul responsable de la situation et la renaissance des émeutes frumentaires traduit la défiance dont il est désormais l'objet. En fait la crise suscite tout à la fois contestations individuelles et séditions collectives, particulièrement vives à Caen où la répression est sévère. Et finalement, les protestations se portent aussi contre l'empereur. Au total, la crise a joué un rôle essentiel dans le détachement des Français à l'égard du régime et de son chef.

Bien des contemporains ont été marqués par la façon dont les conséquences de la campagne de Russie ont accéléré la désaffection des populations à l'égard de l'Empire. Les échos de l'affaire Malet ont été très vite étouffés dans les provinces, on note seulement çà et là quelques 《 clameurs séditieuses $»^{78}$. En revanche la publication du $29^{\mathrm{e}}$ bulletin de la Grande Armée, le 16 décembre, révélant le désastre de la retraite de Russie, marque une nouvelle rupture dans l'histoire du rapport des Français à l'Empire. Le comte de Lavalette indique que les détails contenus dans le $29^{\mathrm{e}}$ bulletin « firent horreur » et ont jeté « la consternation » dans « la France entière $»^{79}$. À l'inquiétude s'ajoute bientôt l'indignation, on observe un peu partout en France que les protestations se font de plus en

(77) Nous nous référons dans ce qui suit aux Bulletins de police. Voir de plus amples précisions dans Les Français et l'Empire.

(78) AN, $\mathrm{F}^{\mathrm{lc}} \mathrm{III} / Y o n n e / 10$, lettres du préfet de l'Yonne au ministre de l'Intérieur, 15 novembre, 23 décembre 1812.

(79) Mémoires et souvenirs du comte de Lavalette (1769-1830), Paris, Le Mercure de France, 1994, p. 277. 
plus nombreuses, révélatrices du divorce qui se consomme alors entre les Français et le régime impérial.

L'année 1814 s'ouvre pour les Français sur l'effroi devant la guerre et sur des désirs de paix. Avec l'invasion du territoire par les armées ennemies, situation inédite depuis les temps révolutionnaires, avec le retour des Bourbons générateur d'une prise de conscience politique nouvelle, avec les Cent-Jours, inspirateurs d'une réinvention du lien au souverain et à la nation, les années 1814-1815 sont un moment clef de l'histoire de la France et de l'Europe du XIX ${ }^{\mathrm{e}}$ siècle. Si l'achèvement de cette période troublée fascine encore pour son $"$ esprit de sacrifice $»^{80}$, si Waterloo prouve qu'une défaite peut après tout être glorieuse ${ }^{81}$, il ne faut pas pour autant lire ces années lourdes de signification au seul prisme de l'héroïsme qu'elles ont révélé. L'attitude des populations en 1814 a fait l'objet d'une approche nouvelle de Jacques Hantraye ${ }^{82}$. Il souligne la relative inertie des populations face à l'envahisseur. Mais aux cas des communes dont les maires n'hésitent pas à engager des pourparlers avec les chefs alliés pour éviter les affrontements, on peut opposer les situations où les civils ne se résignent pas à l'invasion. Les rapports rédigés par les courriers de malles poste prouvent cependant qu'il existe un attachement des populations à l'empereur, sa présence redonne confiance à des civils qui éprouvent le besoin de voir le souverain ${ }^{83}$. Si la violence de guerre a été très lourdement ressentie, les populations ne se sont pas pour autant dressées unanimes devant l'ennemi.

La densité et la nature des événements qui se produisent alors conduisent à une clarification des positionnements des uns et des autres, et pas seulement dans les hautes sphères du pouvoir. Et face aux « girouettes », nombreuses en cet univers-la ${ }^{84}$, bien des anonymes n'hésitent pas alors à clamer leur fidélité, à laisser apparaître au grand jour leur royalisme pour les uns, leur «napoléonisme » pour les autres. À la faveur de ces temps troublés, l'historien dispose d'un observatoire

(80) Dominique de Villepin, Les Cent-Jours ou l'esprit de sacrifice, Paris, Perrin, 2001, $634 \mathrm{p}$.

(81) Jean-Marc Largeaud, Napoléon et Waterloo. La défaite glorieuse de 1815 à nos jours, Paris, La Boutique de l'histoire, 2006, 462 p.

(82) Jacques Hantraye, Les cosaques aux Champs-Elysées. L'occupation de la France après la chute de Napoléon, Paris, Belin, 2005, 303 p.

(83) Jacques Hantraye, «Les formes d'information du pouvoir et les représentations de la guerre au cours de la crise de 1813-1814 en France », dans Natalie Petiteau (dir.), Conflits d'Empire. Cahiers du GERHICO, n 9, 2006, p. 101.

(84) Pierre SERnA, La République des girouettes (1789-1815... et au-delà). Une anomalie politique : la France de l'extrême centre, Seyssel, Champ Vallon, 2005, 570 p. 
d'où se laissent soudain mieux voir les processus d'affirmation de choix politiques qui n'avaient soit pas la possibilité, soit pas la nécessité de s'exprimer depuis 1800 . Le mécontentement contre l'empereur gronde en de nombreux endroits. 1814 est un temps de renaissance de la contrerévolution bien au-delà des rangs étroits des réseaux royalistes. Toute la société française se trouve de nouveau traversée par des appels à faire le choix politique de l'ancienne dynastie. Ce qui n'empêche pas cette contre-révolution renaissante de rencontrer une opposition diffuse plus spontanée. 1814 confirme l'absence d'unanimité y compris face au danger de l'invasion du territoire par les armées étrangères. Si les opposants à l'Empire osent mieux que jamais se montrer à visage découvert, la situation dramatique dans laquelle s'enfonce le pays n'empêche pas l'expression de fidélités sincères à la cause du régime impérial.

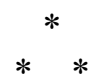

La complexité des attitudes politiques dans les différentes sphères de la société française sous le Consulat et l'Empire apparaît désormais suffisamment pour que l'on abandonne les clichés d'une France totalement hostile à un régime assoiffé de sang ou d'un peuple entièrement dévoué à un souverain né de la Révolution. Dans le monde des élites comme aux échelons plus modestes de la hiérarchie sociale, adhésions et oppositions existent, variables parfois selon les données de la conjoncture économique et militaire, plus ou moins tranchées selon les degrés d'implication dans la vie politique. Exprimées de façon feutrée dans les sphères officielles par quelques élites sociales et politiques, elles éclatent parfois avec vigueur dans des mots de haine ou dans des paroles maladroites de dévotion qui prouvent toutes, en tout cas, que, même parmi les anonymes, une vie politique a subsisté après la Révolution. Les habitudes de prise de parole n'ont pas disparu, la propension à exprimer un avis sur la conduite des affaires de la nation existe encore. Le Consulat et l'Empire ne doivent plus être envisagés comme une page blanche de l'histoire politique de la France puisque même parmi ceux dont la maitrise de la culture écrite est des plus mauvaises, il existe une capacité à s'adresser au souverain ou à faire apparaître son opinion dans l'espace public. Et si l'écrit demeure inaccessible, les mots peuvent jaillir aisément, or s'ils sont entendus par les mouches de la police, ils le sont aussi par d'autres citoyens.

Il reste néanmoins à poursuivre les reconstitutions de trajectoires individuelles et collectives afin de mieux saisir ce qu'ont été les chemine- 
ments politiques des uns et des autres, de part et d'autre des années 18001815, afin de mieux repérer ruptures et continuités au-delà du personnel politique $^{85}$ ou de groupes bien définis comme la noblesse impériale. Des approches régionales enrichissant ce que l'on perçoit déjà pour quelques villes comme Bordeaux, Lille, Sedan ou Elbeuf seraient nécessaires. Des biographies d'élites provinciales seraient bienvenues. Il reste aussi à explorer d'autres espaces spécifiques, il reste également à porter notre attention sur d'autres événements. Les sources sont abondantes dans les provinces autant qu'à Paris, leur abondance même peut décourager, leur nature aussi, puisque souvent produites par le pouvoir. Mais puisque, d'une façon comme d'une autre, les mots d'adhésion et d'opposition existent, il est possible de progresser dans la connaissance de leur signification politique et de leur provenance sociale, donc dans la construction d'une histoire sociale du politique.

Natalie Petiteau

Université d'Avignon

Centre Norbert Elias

74, rue Louis Pasteur

84029 Avignon cedex natalie.petiteau@univ-avignon.fr 